This item was submitted to Loughborough's Research Repository by the author.

Items in Figshare are protected by copyright, with all rights reserved, unless otherwise indicated.

\title{
Model predictions of grain boundary chromium depletion in Inconel 690
}

PLEASE CITE THE PUBLISHED VERSION

PUBLISHER

(C) Elsevier

LICENCE

CC BY-NC-ND 4.0

REPOSITORY RECORD

Yin, Y., and Roy G. Faulkner. 2007. "Model Predictions of Grain Boundary Chromium Depletion in Inconel 690". figshare. https://hdl.handle.net/2134/2817. 
This item was submitted to Loughborough's Institutional Repository by the author and is made available under the following Creative Commons Licence conditions.

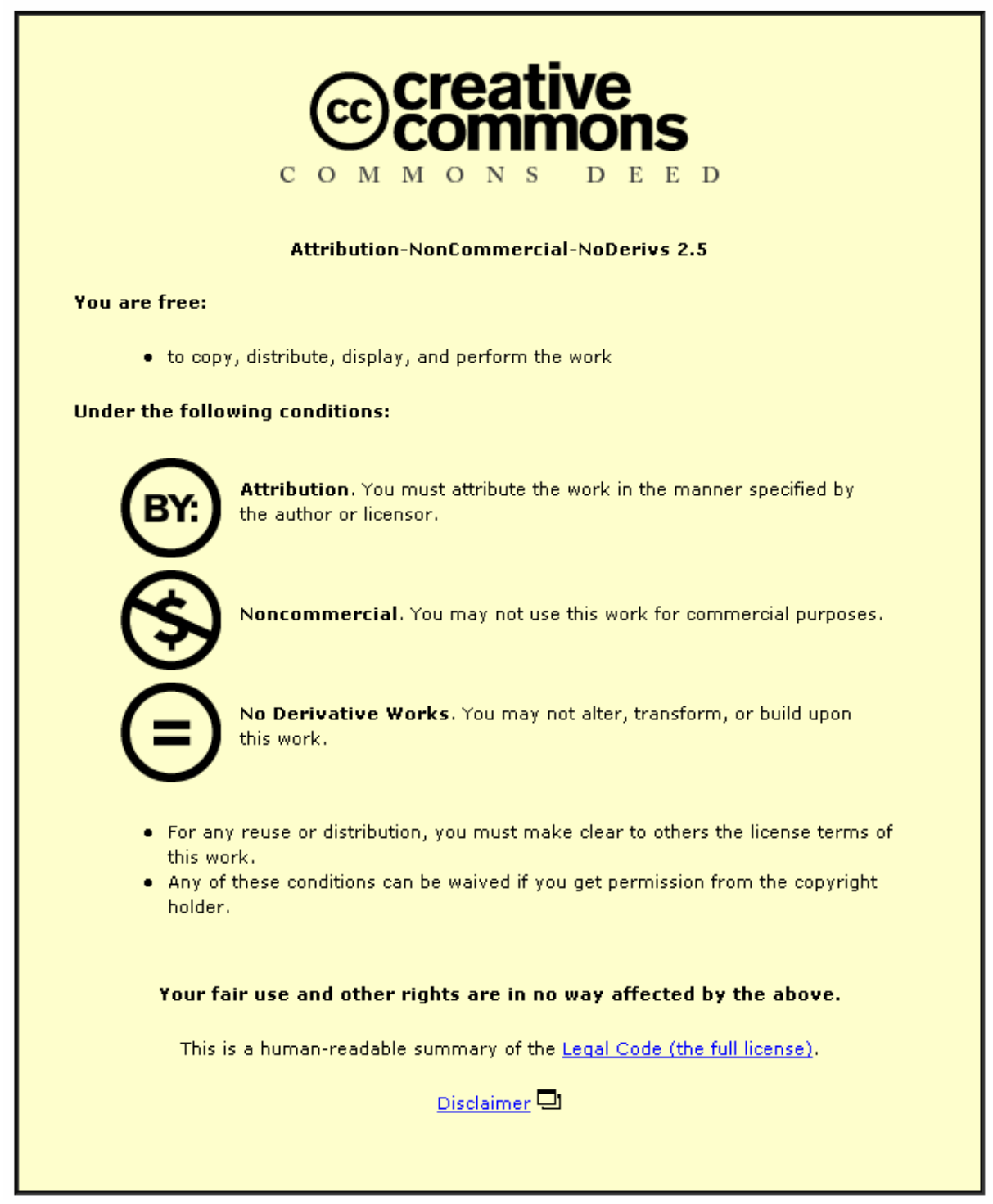

For the full text of this licence, please go to: http://creativecommons.org/licenses/by-nc-nd/2.5/ 


\title{
Model Predictions of Grain Boundary Chromium Depletion in Inconel 690
}

\author{
You Fa Yin*, Roy G. Faulkner \\ IPTME, Loughborough University, Loughborough, Leicestershire, LE11 3TU, UK
}

\begin{abstract}
A new three dimensional modelling technique has been developed to predict chromium depletion from grain boundaries in Ni-Cr-Fe alloys. The technique is based on precipitation kinetics represented by the Zener's Equation and the error function solution of the diffusion law. Unlike former models, the model predicts a natural transition from sensitization to desensitisation and time delay in reaching the minimum chromium concentration at grain boundaries. In addition, the whole prediction can be made using a single Excel worksheet, therefore provides a simple and effective tool for sensitization/desensitisation predictions and studies. Grain size effects and TemperatureTime-Concentration (TTC) maps can also be predicted. The model has been validated against experimental investigations reported in the literature on Inconel 690 aged at four temperatures for different time periods and good agreement between model predictions and experimental observations of chromium depletion has been achieved.
\end{abstract}

Keywords: Alloy (A); Nickel (A); Modelling studies (B); Intergranular corrosion (C); Stress corrosion $(\mathrm{C})$

\section{Introduction}

Chromium and carbon are common alloying elements in many power plant austenitic steels and nickel based alloys. The coexistence of these two elements promotes the

* Corresponding author. Tel: +44(0)1509 223168; Fax: +44(0)1509 $223949 \quad E$ mail addresses: y.yin@lboro.ac.uk (YFY); r.g.faulkner@lboro.ac.uk (RGF) 
formation of chromium-rich carbides, such as $\mathrm{M}_{23} \mathrm{C}_{6}$ and $\mathrm{M}_{7} \mathrm{C}_{3}$, especially in grain boundaries. Under favourable welding and/or heat treatment conditions, the formation of grain boundary chromium-rich carbides sucks chromium from grain boundaries and neighbouring matrix, leaving a chromium depleted zone extending to both sides of the grain boundaries. If the chromium content in the depleted zone is lower than a critical level, it is then vulnerable to environmental corrosion, leading to inter-granular attack (IGA), or inter-granular stress corrosion cracking (IGSCC) $[1,2]$. For these reasons, many studies have been devoted to correlating IGSCC to grain boundary characteristics [3-5] and to a quantitative evaluation of the dechromised zones by experimental analysis and empirical or analytical computer modelling [6-10].

The importance of modelling grain boundary chromium depletion is that a successful model can be used to assist future alloy design and to predict whether or not inter-granular stress corrosion cracking may or may not take place in a specified alloy as a result of a specific heat treatment history. However, there are difficulties in modelling chromium depleted zones near grain boundaries because all alloys are a multi-component systems and grain boundary precipitation kinetics is complex in such a system. It is inevitable therefore that assumptions are made in various models developed over the years [10-15]. A common assumption is that the concentration of carbon is uniform throughout the matrix and grain boundary at any instant. This assumption is reasonable because that diffusivity of carbon is several orders of magnitude higher than that of chromium [10-12]. Under such an assumption, there is as much carbon available as required by grain boundary carbide growth at grain boundaries and therefore carbide nucleation is completed at the instant $t=$ 0 . In such a case, the minimum chromium concentration at grain boundaries is reached at the beginning of precipitation $(t=0)$. As precipitation progresses, the carbon activity 
decreases and chromium concentration increases as required by local equilibrium at the interface. Models by Stawström and Hillert [11], Was and Kruger [10], and Bruemmer [12] have applied this concept and reasonably good agreement with experimental chromium concentration profiles has been obtained. However, experimental observations show that there is a delay in reaching the minimum chromium concentration, i.e. the minimum chromium concentration will only be reached after a finite time rather than at the beginning of precipitate growth $[6,9,16-19]$. Although one may argue that there are errors in the measured grain boundary chromium concentration due to effects such as electron beam broadening in transmission electron microscopy, the width of the chromium depleted zones compared to the size of common spot size used in determination of grain boundary chromium concentration suggest that the delay in reaching the minimum is a real effect. Two points must be considered here. Firstly, in the formation of grain boundary chromium rich carbides, the rate controlling element is chromium rather than carbon as it is commonly accepted that a phase transformation is controlled by the slowest moving atomic species. Therefore, the formation and growth of these carbides takes a relatively long time in order for sufficient chromium atoms to diffuse to grain boundaries and the assumption that nucleation and full growth completes at $t=0$ is not correct. In fact, at lower temperatures, the growth of carbides may not even start at $t=0$ as shown in many temperature-time-transformation diagrams for carbides. Secondly, it is important to realise that we are dealing with para-equilibrium. The thermodynamics dictate that the grain boundary Cr content should be constant at a given temperature, but it takes time for this situation to establish itself. At earlier stages, carbides grow faster and the net result is a decrease of $\mathrm{Cr}$ content. There is a point where carbide growth is sufficiently slow that the amount of $\mathrm{Cr}$ taken by grain boundary carbides is exactly balanced by that diffused to the boundary and the grain boundary $\mathrm{Cr}$ content reaches its minimum. After this point, the $\mathrm{Cr}$ 
diffused to grain boundaries exceeds that taken by the carbides and the net result is an increase in $\mathrm{Cr}$ content as a function of time. The phenomenon was realised many decades ago and is in agreement with the widely accepted depleted-zone theory [20].

In recognition of this problem, Sahlaoui et al. [14] have proposed a new approach based on Mayo's work [13] where the process is divided into two stages. The depletion stage occurs during the process of carbide growth and grain boundary chromium concentration is described by an empirical exponential equation. After the carbide growth is completed, homogenisation of the chromium concentration gradient occurs due to chromium diffusion, and self-healing takes place. Good agreement between the model predictions and experimental measurements on Inconel 690 is achieved. However, as Sourmail et al. [15] have pointed out, the two-stage description of the process violates the reasonable assumption that the interface should remain in local equilibrium during diffusioncontrolled carbide growth. Sourmail et al. [15] recently proposed another approach where a finite-difference model is linked to thermodynamic calculation software and carbon activity and interface composition can be calculated. The authors claim that the approach shows a natural transition from sensitization to desensitization and thus explained the observation of delays in reaching the minimum chromium content. However, the predicted chromium levels are much lower than those observed in experimental measurements.

Here we report our recent development in the prediction of chromium depletion from grain boundaries. The proposed model has the advantages of the model proposed by Sourmail et al. [15], i.e. naturally predicting the transition from sensitisation to desensitisation and delays in reaching the minimum chromium concentration at grain boundaries. In addition, the model gives predictions similar to those predicted by Sahlaoui et al. [14], which are 
therefore, are in good agreement with experimental observations. Grain size effects on chromium depletion and TTC maps can also be predicted using the proposed model.

\section{Model details}

\subsection{The model}

It is widely accepted and has been widely used in previously proposed models that-solute distribution adjacent to grain boundaries can be described by the following error function solution of the diffusion law in a one dimensional approximation [21, 22]:

$$
C=C_{\text {min }}+\left(C_{\text {max }}-C_{\text {min }}\right) \operatorname{erf}\left(\frac{x}{2 \sqrt{D t}}\right) \quad x \leq d / 2
$$

where $d$ is the grain size, $x$ is the distance from grain boundary, $D$ the volume diffusion coefficient, $C$ solute concentration at location $x, C_{\min }$ the solute concentration at grain boundary or the interface between particles and matrix and $C_{\max }$ the solute concentration at the centre of the grain. If a grain can be approximated by a box with sides of $a, b$ and $c$ as shown in Fig. 1, Eqn. (1) can be used with a little modification:

$$
C=C_{\text {min }}+\left(C_{\text {max }}-C_{\text {min }}\right) \operatorname{erf}\left(\frac{x}{2 \sqrt{D t}}\right) \operatorname{erf}\left(\frac{y}{2 \sqrt{D t}}\right) \operatorname{erf}\left(\frac{z}{2 \sqrt{D t}}\right) \quad\left\{\begin{array}{l}
x \leq a / 2 \\
y \leq b / 2 \\
z \leq c / 2
\end{array}\right.
$$

where $x, y$ and $z$ are distances from three adjacent grain boundaries. It is clear that Eqn. (2) is only valid within the smaller cube (shaded in Fig. 1), which is $1 / 8$ of the grain. In all other 7 smaller cubes, the concentration profile can be obtained by translational transformations of Eqn. (2).

If the volume of the grain excluding the grain boundary precipitate particles is $V$ and the average solute concentration in such a volume is $\bar{C}$, then we have, 


$$
\begin{aligned}
\bar{C} V & =\iiint C d V \\
& =C_{\text {min }} V+8\left(C_{\text {max }}-C_{\text {min }}\right) \int_{0}^{a / 2} \operatorname{erf}\left(\frac{x}{2 \sqrt{D t}}\right) d x \int_{0}^{b / 2} \operatorname{erf}\left(\frac{y}{2 \sqrt{D t}}\right) d y \int_{0}^{c / 2} \operatorname{erf}\left(\frac{z}{2 \sqrt{D t}}\right) d z \\
& =C_{\text {min }} V+8 k\left(C_{\text {max }}-C_{\text {min }}\right)
\end{aligned}
$$

where

$$
k=\int_{0}^{a / 2} \operatorname{erf}\left(\frac{x}{2 \sqrt{D t}}\right) d x \int_{0}^{b / 2} \operatorname{erf}\left(\frac{y}{2 \sqrt{D t}}\right) d y \int_{0}^{c / 2} \operatorname{erf}\left(\frac{z}{2 \sqrt{D t}}\right) d z
$$

Note the integration is with respect to $\mathrm{x}, \mathrm{y}, \mathrm{z}$, therefore both $C_{\max }$ and $C_{\min }$ can be regarded as constants. The factor 8 is included because the integration is only carried out in one of the smaller cubes defined in Eqn. (2) and there are 8 of them all together in the grain. The evaluation of the integrals of error functions is well established and the following formula can be used

$$
\int \operatorname{erf}(x) d x=x \operatorname{erf}(x)+\frac{1}{\sqrt{\pi}} \exp \left(-x^{2}\right)
$$

Solving Eqn. (3) for $C_{\min }$, we can obtain

$$
C_{\min }=\frac{\bar{C} V-8 k C_{\max }}{V-8 k}
$$

It is obvious that

$$
V=\left(1-V_{f}\right) V_{0}
$$

where $V_{0}=a b c$ is the total volume of the grain and $V_{f}$ is the volume fraction of the grain boundary precipitates. The average solute concentration can also be determined from $V_{f}$ :

$$
\bar{C}=\left(C_{0}-\frac{V_{f} \rho_{\theta} N_{S}}{\rho_{\alpha}}\right) \frac{1}{1-V_{f}}
$$

where $C_{0}$ is the initial solute concentration (Equals to $C_{\max }$ when the concentration at grain centre is not affected by grain boundary precipitation), $\rho_{\alpha}$ and $\rho_{\theta}$ are densities of the 
matrix and the precipitates respectively, $N_{S}$ is the number of solute atoms in the precipitate molecules ( $N_{S}=23$ for $\mathrm{M}_{23} \mathrm{C}_{6}$ ). Combining Eqns. (6) and (7) yields

$$
\bar{C} V=\left(C_{0}-\frac{V_{f} \rho_{\theta} N_{S}}{\rho_{\alpha}}\right) V_{0}
$$

When the volume diffusion coefficient is known, $k$ can be evaluated for any time $t$ using Eqn. (4) and $C_{\max }$ can be assumed to be constant and equal to the initial concentration $C_{0}$. Therefore it is necessary to estimate the volume fraction of the precipitates in order to determine $C_{\min }$ using Eqn. (5).

It is commonly accepted and supported by experimental measurements that the evolution of precipitate size, $r$, follows Zener's relation during the growth stage [17]:

$$
r \propto \sqrt{t}
$$

In such a case, the volume fraction of precipitates obeys the relation

$$
V_{f} \propto t^{3 / 2}
$$

as $V \propto r^{3}$. Therefore, an equation is constructed to describe the evolution of precipitate volume fraction:

$$
V_{f}=V_{f, \max } \frac{t^{3 / 2}}{t^{3 / 2}+\alpha t_{\max }^{3 / 2}}
$$

Where $V_{f, \max }$ is the maximum volume fraction or volume fraction at equilibrium, $\alpha$ is a constant which determines the volume fraction when $t=t_{\max }$. It is clear that Eqn. (11) is equivalent to Eqn. (10) when $t^{3 / 2}<<\alpha t_{\max }^{3 / 2}$ and gives $V_{f}=V_{f, \max }$ when $t \rightarrow \infty$. Some plots of Eqn. (11) with $\alpha=0.57\left(V_{f}=0.64 V_{f, \max }\right.$ when $\left.t=t_{\max }\right)$ and $t_{\max }=0.5,5,50$ and 
$120 \mathrm{~h}$ are shown in Fig. 2, together with results produced using a Monte Carlo based precipitation kinetic simulation $[23,24]$ of Inconel 690 at 538, 600, 700 and $800{ }^{\circ} \mathrm{C}$. It is evident that Eqn. (11) describes the evolution of precipitate volume fraction reasonably well, especially for the shorter time periods where the main process is particle growth.

The maximum volume fraction of grain boundary precipitates can be estimated from the concentrations and the molecular formula of the precipitate using the following equation [25]:

$$
V_{f, \max }=\frac{\rho_{\alpha}\left(C_{C}-C_{C, E}\right)}{N_{C} \rho_{\theta}}
$$

where $C_{C}$ is the initial carbon concentration, $C_{C, E}$ is the solubility of carbon and $N_{C}$ is the number of carbon atoms in the molecular formula of the precipitates (e.g. in $\mathrm{M}_{23} \mathrm{C}_{6}$, $\left.N_{C}=6\right) . C_{C, E}$ can be calculated using thermodynamic calculation software such as MTDATA $[26,27]$. However, in most cases, $C_{C, E}<<C_{C}$ and can be neglected. The maximum volume fraction can also be calculated using masses of the phases from MTDATA calculations.

\subsection{Material}

The material used to test the model is Inconel 690, which is a Ni base alloy and is used in power generation industries. The reason for us to choose this material is that Kai e. al. [2830] have studied the microstructural evolution and chromium depletion profiles of the material in detail and so it is convenient use the data to validate our models. The composition of it is shown in Table 1. Details of heat treatment of the material can be found in reference [28]. 


\subsection{Modelling parameters}

The whole modelling process is carried out using a Microsoft Excel Worksheet with a few simple macros to carry out some calculations, such as the volume fraction of grain boundary precipitates. The input parameters required are listed in the Table 2. Most of the parameters are easy to be found or estimated, except $t_{\max }, \alpha$ and diffusion coefficient $D$. Three parameters, $a, b, c$, are used to describe the dimensions of the grains in the material. Here a value of $24 \mu \mathrm{m}$ is used for all three dimensions as this is the average grain size reported by Kai et al. [28]. Concentrations and solubility of elements of concern can be input either in mole fractions or weight percent. Concentration is specified in the composition. Solubility can be calculated using MTDATA $[25,26]$ and is a function of temperature and composition of the material. As the solubility of carbon is very small as compared to the concentration, its effects on the results are also small. The molar densities of the matrix and the precipitate phase are estimated from their densities. $N_{S}$ and $N_{C}$ are determined by the grain boundary precipitates. The listed values in Table 2 are for $\mathrm{M}_{23} \mathrm{C}_{6}$.

From Eqn. (11), $t_{\max }$ and $\alpha$ are independent. It has been found that $t_{\max }$ equals roughly the time required to reach the minimum grain boundary chromium concentration if $\alpha$ is set to 0.57 for different temperatures. Although Kai et al. [28] and Sahlaoui et al. [14] used diffusion coefficients of chromium in Inconel 600 measured by Pruthi et al. [31] at temperatures above $800{ }^{\circ} \mathrm{C}$ and extrapolated to lower temperatures by Borello et al. [32], we found that these diffusion coefficients do not work well here. Was and Kruger [10] also found that these diffusion coefficients had to be adjusted to match their predictions with experimental measurements on a Ni-16Cr-9Fe alloy and the fitted diffusion coefficients were higher than those reported by Pruthi et al. We also did some analysis of Cr depletion 
profiles of Inconel 600 and found that these diffusion coefficients are applicable at $800{ }^{\circ} \mathrm{C}$, but higher diffusion coefficients had to be used at lower temperatures. Cr content in Inconel 690 is much higher than that in Inconel 600; therefore the diffusion coefficients are different. As there are no experimental data on diffusion coefficients for $\mathrm{Cr}$ in Inconel 690, we used a similar approach to Was and Kruger [10] and treat the diffusion coefficients as adjustable parameters. Ideally, the precipitation kinetics here should be treated rigorously as a multicomponent diffusion problem $[33,34]$. In effect, we have already considered the multi-component effect in equation (12), where the effect of other elements on the volume fraction of chromium carbide is considered using the thermodynamic data bank MTDATA $[26,27]$. This software automatically allows for the effect of other elements on the thermodynamic stability of the carbide phase. Higher volume fractions imply deeper depletion effects near to the boundary, with a resultant overall lower value of $\mathrm{c}$ min. Another approach that is currently being considered, but not used in this paper, is to modify the thermodynamically predicted equilibrium minimum chromium concentration given in Eqn. (5), making allowance for the multicomponent nature of the material. This can be done by considering the thermodynamics of the chromium carbide phase in a multicomponent ferrous alloy system using thermodynamic data banks, e.g. MTDATA [26,27 35]..

\section{Results and Discussion}

\subsection{Chromium Concentration Profiles}

Figs. 3-6 show some examples of chromium concentration profiles adjacent to grain boundaries in Inconel 690 aged at 538, 600, 700 and $800^{\circ} \mathrm{C}$ for different durations. In all these graphs, squares are experimental measurements from [28] and lines are predictions from this work. As mentioned before, $\alpha=0.57$ is used for all temperatures and durations. 
However, $t_{\max }$ and diffusion coefficients vary considerably with temperature and the values used are listed in Table 3 . The grain boundary concentration is corrected for beam broadening effects using a simple approach by Faulkner et al. [36],

$$
C_{\min }^{\prime}=C_{\min }^{M}-\frac{2 \sigma m}{\sqrt{2 \pi}}
$$

where $C_{\min }^{\prime}$ and $C_{\min }^{M}$ are the corrected and measured grain boundary concentration respectively, $\sigma$ is the slope of profile at grain boundaries and $m$ is the spot size. The spot size is $20 \mathrm{~nm}$ as reported by Kai et al. [28]. As the spot size for all measurements is the same, the corrections are determined by the gradient of the concentration profile at the vicinity of grain boundaries. A steeper profile results in a larger correction. The corrected grain boundary chromium concentrations are represented by circles in Figs. 3-6. The predictions are made using the parameters in Tables 2 and 3.

From Figs. 3-6, the corrections made to the grain boundary chromium concentration are significant in most cases, especially in the cases of short ageing time and lower temperatures where the concentration profile gradients are relatively steep. The corrections also vary from case to case due to the difference in the gradients. For example, the minor correction at $24 \mathrm{~h}$ in Fig 3 is due to the nearly flat profile. The grain boundary $\mathrm{Cr}$ concentration at $100 \mathrm{~h}$ is much lower than that at $24 \mathrm{~h}$, therefore gives a much higher slope, though the profile is also wider. Note the temperature corresponding to this figure is the lowest. At higher temperatures, opposite situations may occur. For example, Figure 4 shows a much more substantial correction at $24 \mathrm{~h}$ than that at $100 \mathrm{~h}$. This illustrates the importance of making corrections before comparing model predictions and experimental measurements. 
Figs. 3-6 also show that the predictions made in this work are in very good agreement with experimental measurements at all four temperatures and for most ageing durations. There are some predicted profiles that deviate from the experimental measurements. As only a few grain boundaries were examined in determining the concentration profiles and we know that grain size has significant effects on the evolution of the concentration profile (see sections below), the deviations can be attributed mainly to experimental uncertainties.

\subsection{Effect of Temperature}

Figs. 3-6 demonstrated the evolution of the chromium concentration profile as a function of temperature. The sensitization and desensitization process is more clearly demonstrated by plotting grain boundary chromium content as a function of ageing time. Figs. 7-10 show such plots for the four temperatures studied. The squares in the graphs are measured data from reference [28], the circles are the corrected values of the measurements according to Eqn. (13) and lines are predictions from this work. In general, the predicted grain boundary chromium content is in good agreement with measurements for all four temperatures.

As it is expected, Figs. 7-10 show that ageing temperature has significant effects on the sensitization and desensitization process. The lower the ageing temperature, the slower the depletion and the lower the minimum chromium concentration. The self-healing process starts earlier at higher temperatures.

It is well understood that the sensitization and desensitization process is closely related to the evolution of carbon concentration in the matrix and the amount of carbon in solid solution itself also has effects on the IGSCC resistance of alloys [37]. The concentration of carbon in the matrix can be predicted by the present model and Fig. 11 shows the carbon 
content as functions of ageing time and temperature. At lower temperatures the carbon content in the matrix decreases very slowly during the initial stages of ageing, where nucleation takes place and particles grow slowly. This corresponds to a very slow increase in the volume fraction of the precipitates as shown in Fig. 2. As a result, grain boundary chromium concentration decreases slowly at lower temperatures (Figs. 7-10). When the particles are larger, the volume fraction of particles increases sharply and results in a steep decrease in matrix carbon content. The decrease of carbon content continues until it reaches a level very close to its solubility at the ageing temperature, where the volume fraction of the particles is very close to its maximum (Fig. 2).

\subsection{Effect of Grain Size}

Yu and Yao [8] and Kai et al. [28] have calculated the effects of grain size on the chromium concentration profile in Inconel 690 aged at $700{ }^{\circ} \mathrm{C}$ for $48 \mathrm{~h}$ using a modified version of the model by Was and Kruger [10]. Sourmail et al. also studied this effect [15]. In both cases, significant effects have been found. The effect has been studied here by considering $t_{\max }$ in Eqn. (11) as a function of grain size.

The total volume of grain boundary precipitate particles, $V_{P}$, can be calculated using Eqn. (11) if the volume fractions are replaced by the total volume:

$$
V_{P}=V_{P, \max } \frac{t^{3 / 2}}{t^{3 / 2}+\alpha t_{\max }^{3 / 2}}
$$

If the total grain boundary area is $A$, then the volume of precipitate particles per unit grain boundary area is

$$
V_{A}=\frac{V_{P}}{A}=\frac{V_{P, \max }}{A} \frac{t^{3 / 2}}{t^{3 / 2}+\alpha t_{\max }^{3 / 2}}
$$


As volume fractions of grain boundary precipitate particles are independent of grain size (see Eqn. (12)) if all the precipitates are in grain boundaries, the maximum total volume of grain boundary precipitates is directly proportional to the volume of the grain, which in turn is directly proportional to the product of grain boundary area, $A$, and grain size, $d$. Therefore,

$$
\frac{V_{P, \max }}{A}=\beta d
$$

and

$$
V_{A}=\beta d \frac{t^{3 / 2}}{t^{3 / 2}+\alpha t_{\max }^{3 / 2}}
$$

where $\beta$ is a constant determined by composition of the material, temperature and geometry of the grain. When $t \rightarrow 0$, we have

$$
V_{A} \approx \beta d \frac{t^{3 / 2}}{\alpha t_{\max }^{3 / 2}}
$$

The precipitate volume per unit area of grain boundary calculated using Eqn. (18) should be the same for different grain sizes as this is only affected by solute diffusion very close to the grain boundaries in such a short time and therefore is independent on grain size. Using a reference grain size, $d_{0}$ and the corresponding time, $t_{\max , 0}$ at the same temperature, we have

$$
\beta d \frac{t^{3 / 2}}{\alpha t_{\max }^{3 / 2}}=\beta d_{0} \frac{t^{3 / 2}}{\alpha_{0} t_{\max , 0}^{3 / 2}}
$$

Because $\alpha$ and $t_{\max }$ are independent, $\alpha$ can be kept as constant to ensure that $t_{\max }$ corresponds the time to reach the minimum grain boundary concentration. Under such conditions, the following equation can be derived from Eqn. (19):

$$
t_{\max }=\left(\frac{d}{d_{0}}\right)^{2 / 3} t_{\max , 0}
$$


This equation is used here to study the effects of grain size with $d_{0}=24 \mu \mathrm{m}$.

Fig. 12a presents the predicted effects of grain size on the evolution of grain boundary chromium content at $700{ }^{\circ} \mathrm{C}$ and Fig. $12 \mathrm{~b}$ shows the effects of grain size on the chromium concentration profile in Inconel 690 aged at $700{ }^{\circ} \mathrm{C}$ for $48 \mathrm{~h}$ using the model proposed in this work. As both figures show, the effects of grain size are very significant. Larger grains result in much wider chromium depleted zones and much lower minimum grain boundary chromium concentrations, and consequently much lower IGSCC resistance. On the other hand, smaller grain sizes will result in faster kinetics, similar to the effects of increasing temperature, and give rise to an increase of minimum grain boundary chromium content. For small grains, the volume of grain boundary carbides is limited by the carbon content inside the grain, i.e. the amount of chromium taken from adjacent matrix is limited. For larger grains, there is more carbon available. The volume of grain boundary carbides is higher and normally they grow to larger sizes. This means that more $\mathrm{Cr}$ is taken from the adjacent matrix. On the other hand, $\mathrm{Cr}$ needs to diffuse longer distances to reach the grain boundary. Therefore, the process takes a longer time and the minimum $\mathrm{Cr}$ content is lower (because the growth of carbides continues taking $\mathrm{Cr}$ from the matrix).

The effect of grain size on the chromium concentration profile of Inconel 690 aged at 700 ${ }^{\circ} \mathrm{C}$ for $48 \mathrm{~h}$ in Fig. $12 \mathrm{~b}$ predicted in this work is much more marked than that calculated by Yu and Yao [8] and by Kai et al. [28]. Furthermore, the calculated minimum grain boundary $\mathrm{Cr}$ concentration could be lower than the equilibrium concentration predicted by MTDATA for large grain sizes and lower temperatures. This is attributed to the fact that intra-granular precipitation is not included in the calculation. Intra-granular $\mathrm{M}_{23} \mathrm{C}_{6}$ particles nucleated at dislocation lines in Inconel 690 have been observed [28]. Therefore, it is unrealistic to exclude intra-granular precipitation from $\mathrm{Cr}$ depletion predictions as intra- 
granular particles share a considerable amount of the available carbon and thus affect the $\mathrm{Cr}$ depletion process on grain boundaries. In other words, intra-granular precipitation of $\mathrm{M}_{23} \mathrm{C}_{6}$ particles decreases the maximum grain boundary particle volume fraction and this effect increases with increasing grain size. To take this into consideration, Eqn. (11) must be modified. The basic considerations are (1) at short times, the grain boundary precipitate volume is not affected by grain size, as discussed above; (2) at sufficiently long times, the ultimate volume fraction of grain boundary particles is reduced by increasing grain size due to increasing proportion of intra-granular precipitates. Statistically speaking, the volume of intra-granular particles is proportional to the volume of the grain, therefore is proportional to the cube of the grain size. On the other hand, the volume of grain boundary particles is proportional to the grain boundary area and therefore is proportional to the square of grain size if the volume of particles per unit area of grain boundary remains the same for different grain sizes. Under these considerations, a further parameter is introduced and Eqn. (11) is modified to

$$
V_{f}=V_{f, \max } \frac{t^{3 / 2}}{\left(\frac{d}{d_{0}}\right)^{n} t^{3 / 2}+\alpha t_{\max }^{3 / 2}}
$$

where $n$ is the new parameter. The value of $n$ for $d>d_{0}$ can be determined using $\mathrm{Cr}$ solubility in the material found in the literature or calculated using MTDATA. Here we use the results of MTDATA calculations. The actual procedure is as follows. Firstly $24 \mu \mathrm{m}$ is used as the reference grain size as before. Then grain size (here all three sides $a, b$ and $c$ ) is set to a very large value $(1000 \mu \mathrm{m})$. Finally adjust $n$ to obtain a minimum grain boundary $\mathrm{Cr}$ concentration that is close to but greater than the solubility calculated using MTDATA. In this study, $n=0.85$ was obtained and interestingly this value seems to work for all temperatures. When $d \leq d_{0}, n$ is set to zero. This implies that the volume of intra- 
granular precipitate and therefore the effect of intra-granular precipitation on $\mathrm{Cr}$ depletion can be neglected when the grain size is small.

The results of the modified effects of grain size on Cr depletion are presented in Fig. 13. Comparison of Fig. 12b and Fig. 13a shows that the effect is reduced considerably by the modification. This implies that intra-granular precipitation has significant effects on $\mathrm{Cr}$ depletion from grain boundaries.

Fig. 13b shows the minimum grain boundary $\mathrm{Cr}$ concentration as a function of grain size and temperature, together with the solubility calculated using MTDATA. The minimum grain boundary chromium concentration increases with increasing temperature, but decreases with increasing grain size. The grain size effects are very significant at small grain sizes, but then decrease as grain size increases and the minimum chromium concentration approaches the chromium solubility predicted using MTDATA.

\subsection{Temperature-Time-Concentration Map}

The generation of the Temperature-Time-Concentration (TTC) map involves the determination of diffusion coefficients and $t_{\max }$ as a function of temperature. Here the relationships were determined from the values of both parameters at the four temperatures studied by simple fitting. The grain size was kept as $24 \mu \mathrm{m}$. The results are shown in Figs. 14. The temperature range in Fig. 14 is from 500 to $800{ }^{\circ} \mathrm{C}$ and time scale is from 0.01 to $1000 \mathrm{~h}$. There is no experimental TTC or Temperature-Time-Sensitisation (TTS) data available to compare with the predictions made here. However, the contour shown in Fig. $14 \mathrm{~b}$ is quantitatively in good agreement with experimental results on stainless steels reported in the literature, such as by Brummer [12] and Strawström and Hillert [11]. 


\section{Conclusions}

Chromium concentration evolution at grain boundaries resulting from inter-granular carbides precipitation during aging of the $\mathrm{Ni}-\mathrm{Cr}-\mathrm{Fe}$ alloys can be predicted with reasonably good accuracy by a model based on Zener's description of grain boundary precipitation kinetics and the simple and well known error function solution of the diffusion law. Unlike former models, the model proposed here predicts a natural transition from the states where grain boundary chromium concentration decreases with increasing heat treatment time to the states where chromium concentration increases with time, i.e. from depletion to self-healing, when the carbides growth is sufficiently slow. A time delay of reaching the minimum chromium concentration at grain boundaries is also naturally predicted. Multi-component effects are also partly considered by using solubility data of chromium and carbon from MTDATA calculations where all elements in the materials are included and interactions are considered. The model is truly three dimensional rather than one dimensional as is the case in most former models and therefore the predictions of chromium depletion and self-healing are more realistic. The effects of Intra-granular carbide precipitation and grain size on grain boundary $\mathrm{Cr}$ depletion are accounted for by the model. Temperature-Time-Concentration diagrams can also be generated with little difficulty. The whole model uses a single Excel worksheet and is easily applicable to different materials and carbides.

Application of the model to Inconel 690 heat treated for different periods of time $(1 \mathrm{~h} \mathrm{to}$ $215 \mathrm{~h}$ ) and four temperatures $\left(538^{\circ} \mathrm{C}, 600^{\circ} \mathrm{C}, 700^{\circ} \mathrm{C}\right.$ and $\left.800^{\circ} \mathrm{C}\right)$ show that good predictions can be made using the model of both $\mathrm{Cr}$ concentration profiles near grain boundaries and the evolution of grain boundary $\mathrm{Cr}$ content. The results show that selfhealing occurs when grain boundary carbide precipitate volume fraction reaches about 
$64 \%$ of its maximum. Sensitisation and desensitisation processes are affected greatly by the grain size and temperature. Lower temperatures and larger grains result in lower minimum grain boundary $\mathrm{Cr}$ content, with resultant lower resistance to inter-granular stress corrosion cracking susceptibility.

\section{Acknowledgement}

The authors would like to thank DTI (Department of Trade and Industry) for funding this work.

\section{References}

1. G. S. Was, and V. B. Rajan, Metall. Trans. 18A (1987) 1313-1323.

2. C. L. Briant, C. S. O. Toole, and E. L. Hall, Corrosion 42 (1986) 15-27.

3. P. Lin, G. Palumbo, U. Erb, and K.T. Aust, Scripta Metallurgica et materialia 33 (1995) 1387-1392.

4. Hong Yun Bi, Hiroyuki Kokawa, Zhan Jie Wang, Masayuki Shimada, and Yutaka S. Sato, Scripta Materialia 49 (2003) 219-223.

5. D. N. Wasnika,V. Kainb, I. Samajdara, B. Verlindenc, P.K. De, Acta Materialia 50 (2002) 4587-4601.

6. E. P. Butler, and M. G. Burke, Acta metall. 34 (1984) 557-570.

7. G.E.C. Bell, P.F. Tortorelli, E.A. Kenik and R.L. Klueh, Journal of Nuclear Materials 179-181 (1991) 615-618.

8. G-P. Yu and H-C. Yao, Corrosion 46 (1990) 391-402.

9. J. J. Kai, C. H. Tsai, T. A. Huang, and M. N. Liu, Metallurgical Transactions A 20 (1989)1077-1088.

10. G. S. WAS and R. M. KRUGER, Acta metall. 33 (1985) 841-854. 
11. C. Stawström, and M. Hillert, Journal of the Iron and Steel Institute 207 (1969) 77-85.

12. S. M. Bruemmer, Corrosion 46 (1990) 698-709.

13. Williams E. Mayo, Materials Science and Engineering A 232 (1997)129-139.

14. H. Sahlaouia, H. Sidhoma, and J. Philibert, Acta Materialia 50 (2002)1383-1392.

15. T. Sourmail, C. H. Too, and H. K. D. H. BHADESHIA, ISIJ International 43 (2003)1814-1820.

16. G. S. Was, H. H. Tischner adn R. M. Latanision, Metallurgical Transactions A, 12A (1981) 1397-1408.

17. E. L. Hall and C. L. Briant, Metallurgical Transactions A, 16A (1985) 1225-1236.

18. H.Sahlaoui, K.Makhlouf, H.Sidhom, J.Philibert, Materials Science and Engineering A372 (2004) 98-108.

19. S. M. Bruemmer and L. A. Charlot: Scr. Metall., 20 (1986), 1019.

20. R. STICKLER and A. VINCKIER: Trans. ASM, 54 (1961) 362.

21. J. Crank, The Mathematics of Diffusion, Oxford University Press, Oxford, 1956.

22. C. Zener, J. Appl. Phys. 20 (1949) 950-953.

23. Y. F. Yin, and R. G. Faulkner, Materials Science and Technology, 19 (2003) 91-98.

24. Y. F. Yin, and R. G. Faulkner, Power Technology, J. Lecomte-Beckers et. al. (eds.), 19(II) (2002) 1247-1256.

25. Y. F. Yin and R. G. Faulkner, Materials Science and Engineering A 344 (2003) 92102.

26. R. H. Davies, A.T. Dinsdale, J.A. Gisby, J.A.J. Robinson, and S.M. Martin, CALPHAD 26 (2002) 229-271.

27. R. H. Davies, A. T. Dinsdale, T. G. Chart, T. I. Barry, and M. H. Rand, High Temperature Science, 26 (1989) 251-262. 
28. J.J. KAI, G.P. YU, C.H. TSAI, M.N. LIU, and S.C. YAO, Metallurgical Transactions A 20 (1989) 2057-2067.

29. J.J. Kai and M.N. Liu, Scripta Metallurgica 23 (1989) 17-22.

30. J.J. Kai, C.H. Tsai and G.P. Yu, Nuclear Engineering and Design 144 (1993) 449-457.

31. D. D. Pruthi, M. S. Anand and R. P. Agarwala, J. Nucl.Mater., 64 (1977), 206-210.

32. A. Borello, S. Casadio, A. Saltelli, and A. Scibona, Corrosion, 37 (1981), 498-505.

33. D. E. Coates, Metall. Trans. 3(1972) 1203-1212.

34. D. E. Coates, Metall. Trans. 4(1973) 395-396.

35. R. G. Faulkner, Y. F. Yin, J. Cintas, and J. M. Montes, in Proceedings of the $12^{\text {th }}$ International Conference on Environmental Degradation of Materials in Nuclear Power System-Water Reactors- TMS (The Minerals, Metals \& Materials Society), Salt Lake City, USA, 2005, pp. 135-142.

36. R. G. Faulkner, T. S. Morgan, and E. A. Little, X-RAY SPECTROMETRY 23 (1994) 195-202.

37. J.L.Hertzberg and G. S.Was, Scripta Metallurgica et Materialia 33 (1995) 1193-1199. 


\section{CAPTIONS TO TABLES AND FIGURES}

Table 1. Composition of Inconel 690 (from ref. [23], wt \%, Ni balance).

Table 2. Required parameters for the model, values are for $T=600{ }^{\circ} \mathrm{C}$.

Table 3. Diffusion coefficients and $t_{\max }$ values for different temperatures.

Figure 1. A schematic three dimensional representation of a grain.

Figure 2. Some examples of precipitate volume fraction as a function of time as compared with simulation results.

Figure 3. Cr concentration as a function of distance from grain boundaries at $538{ }^{\circ} \mathrm{C}$ for different time durations.

Figure 4. Cr concentration as a function of distance from grain boundaries at $600{ }^{\circ} \mathrm{C}$ for different time durations.

Figure 5. Cr content as a function of distance from grain boundaries at $700{ }^{\circ} \mathrm{C}$ for different time durations.

Figure 6. Cr concentration as a function of distance from grain boundaries at $800{ }^{\circ} \mathrm{C}$ for 1 and 10 hours.

Figure 7. Grain boundary $\mathrm{Cr}$ concentration as a function of time at $538{ }^{\circ} \mathrm{C}$.

Figure 8. Grain boundary $\mathrm{Cr}$ concentration as a function of time at $600{ }^{\circ} \mathrm{C}$.

Figure 9. GB Cr concentration as a function of time at $700{ }^{\circ} \mathrm{C}$.

Figure 10. GB $\mathrm{Cr}$ concentration as a function of time at $800{ }^{\circ} \mathrm{C}$.

Figure 11. Calculated carbon concentration in the matrix as a function of time.

Figure 12. Grain size effects on grain boundary $\mathrm{Cr}$ depletion. (a) The effects of grain size on the evolution of minimum grain boundary $\mathrm{Cr}$ concentration at $700{ }^{\circ} \mathrm{C}$; (b) The effects of grain size on the $\mathrm{Cr}$ concentration profile at $700{ }^{\circ} \mathrm{C}$ for $48 \mathrm{~h}$.

Figure 13. Effects of grain size on Cr depletion modified to take intra-granular precipitation into consideration. (a) grain size effect on $\mathrm{Cr}$ concentration profiles near grain boundaries at $700{ }^{\circ} \mathrm{C}$ for $48 \mathrm{~h}$. (b) grain size effect on the minimum grain boundary $\mathrm{Cr}$ concentration as a function of temperature, together with solubility calculated using MTDATA. 
Figure 14. Temperature-Time-Concentration diagrams of Inconel 690. 